\title{
Infrared Thermography with High Accuracy in a Neonatal Incubator
}

\author{
Keisuke Hamada (10, ${ }^{1,2}$ Eiju Hirakawa, ${ }^{3}$ Hidetsugu Asano, ${ }^{4}$ \\ Hayato Hayashi, ${ }^{4}$ Takashi Mine, ${ }^{2,5}$ Tatsuki Ichikawa, ${ }^{2,6}$ \\ and Yasuhiro NAGATA $2,7,8$

\begin{abstract}
${ }^{1}$ Department of Clinical Engineering, Nagasaki Harbor Medical Center, Nagasaki, Nagasaki, Japan; ${ }^{2}$ Department of Comprehensive Community Care Systems, Nagasaki University Graduate School of Biomedical Sciences, Nagasaki, Nagasaki, Japan; ${ }^{3}$ Department of Neonatology, Kagoshima City Hospital, Kagoshima, Kagoshima, Japan; ${ }^{4}$ Research \& Development Group, Technical Department, Atom Medical Corporation, Saitama, Saitama, Japan; ${ }^{5}$ Department of Clinical Oncology, Nagasaki Harbor Medical Center, Nagasaki, Nagasaki, Japan; ${ }^{6}$ Department of Gastroenterology, Nagasaki Harbor Medical Center, Nagasaki, Nagasaki, Japan; ${ }^{7}$ Department of Community Medicine, Nagasaki University School of Biomedical Sciences, Nagasaki, Nagasaki, Japan; and ${ }^{8}$ Leading Medical Research Core Unit, Nagasaki University Graduate School of Biomedical Sciences, Nagasaki, Nagasaki, Japan
\end{abstract}

(Received 17 December 2021; accepted 17 February 2022; published online 2 March 2022)

Associate Editor Umberto Morbiducci oversaw the review of this article.

\begin{abstract}
As the accuracy of body temperature measurement is especially critical in premature infants on admission to the neonatal intensive care unit (NICU), noninvasive measurement using infrared thermography (IRT) has not been widely adopted in the NICU due to a lack of evidence regarding its accuracy. We have established a new calibration method for IRT in an incubator, and evaluated its accuracy and reliability at different incubator settings using a variabletemperature blackbody furnace. This method improved the accuracy and reliability of IRT with an increase in percentage of data with mean absolute error (MAE) $<0.3{ }^{\circ} \mathrm{C}$ to $93.1 \%$ compared to $4.2 \%$ using the standard method. Two of three IRTs had MAE $<0.1{ }^{\circ} \mathrm{C}$ under all conditions examined. This method provided high accuracy not only for measurements at specific times but also for continuous monitoring. It will also contribute to avoiding the risk of neonates' skin trouble caused by attaching a thermistor. This study will facilitate the development of novel means of administering neonatal body temperature.
\end{abstract}

Keywords-IRT, Premature infants, Calibration, Non-invasive, Monitoring, Thermoregulation.

\section{ABBREVIATIONS}

FOV Field of view

NEC Neonatal necrotizing enterocolitis

NIRT Neonatal infrared thermography

FPA Focal plane array

NUC Non-uniformity correction

ETRS External temperature reference source

NETD Noise equivalent temperature difference

SiTF Signal transfer function
Address correspondence to Keisuke Hamada, Department of Clinical Engineering, Nagasaki Harbor Medical Center, Nagasaki, Nagasaki, Japan. Electronic mail: hamada_keisuke@icloud.com

\section{INTRODUCTION}

Body temperature is an important health indicator in the formulation of a clinical management plan, and appropriate management of body temperature is required for maintenance of normal physiological functions and to facilitate prompt recovery after illness. ${ }^{28,} 38$ Especially stringent body temperature management is required for premature infants, and it has been known since the 1960s that reducing heat loss in the first few days of life can improve the survival rate 
of premature infants. ${ }^{36}$ Newborn infants lose heat at a rate two to three times higher than adults, and without treatment, neonatal body temperature usually decreases by $0.1-0.3^{\circ} \mathrm{C}$ per minute..$^{4,41}$

The importance of controlling body temperature in clinical practice has been widely acknowledged since the mercury thermometer was first used for medical purposes in the late 18 th century. ${ }^{42}$ Since the 1980 s, predictive thermometers have commonly been used to measure temperature in newborn infants. Moreover, as neonates require strict body temperature management, reliable and sustainable measurements are necessary using a thermistor probe. However, the insufficiently keratinized epidermal barrier of premature skin makes the attachment of sensors to the body difficult, resulting in inaccurate body temperature measurements. Therefore, there have been a number of clinical and laboratory trials to examine various noninvasive methods for measurement of body temperature. The infrared thermometer is already used as a noninvasive means of measuring body temperature, not only in daily clinical practice but also for non-medical purposes at home. ${ }^{30}$ Infrared thermography (IRT) is a similar method that can be used to determine the temperature of an object by measuring infrared emissions. As IRT has a wide field of view (FOV), it can simultaneously measure multiple objects, and is therefore useful for screening at airports. ${ }^{9,} 13$ For example, IRT has been used for fever screening in the coronavirus disease 2019 (COVID-19) pandemic. 5,8 However, IRT must be used in combination with a Food and Drug Administration (FDA)-approved thermometer due to its low level of accuracy. ${ }^{11}$

IRT was first applied in medical research at Middlesex Hospital in London and the Royal National Hospital for Rheumatic Diseases in Bath, both in the UK, from 1959 to $1961 .^{32}$ In addition, IRT has been used to analyze changes in body temperature due to exercise. $^{15,18,43}$ The medical application of IRT in neonates was first examined in the 1970s. Pomerance et $a l .{ }^{29}$ reported that the skin directly above highly vascular organs, such as the heart, liver, and kidneys, has a higher body surface temperature compared to other areas. Abbas et al. ${ }^{1}$ reported that respiratory rate can be monitored by IRT using a method based on the difference between inspiratory and expiratory nostril temperature. Knobel et $\mathrm{al}^{22}$ reported that abdominal temperature is lower in patients with neonatal necrotizing enterocolitis (NEC) compared to in those without evidence of this condition. Furthermore, both abdominal and leg temperatures of neonates at gestational age 23-28 weeks measured by IRT over the first week after birth were reported to be similar to those measured using a thermistor-based contact method. ${ }^{23}$

For use in clinical practice, methods for measurement of body temperature require a high degree of accuracy due to the critical importance of body temperature management. This is especially true in newborn infants. However, IRT generally has insufficient accuracy for medical use, and it is therefore necessary to improve the accuracy of neonatal infrared thermography (NIRT) to measure the body temperature of newborn infants. Abbas et al. ${ }^{2}$ suggested that different temperature compensation equations should be used according to the measurement environment, i.e., convective neonatal incubators, kangaroo mother care, and open radiant warmers.

There have been no previous reports of IRT installed inside the incubator, with previous studies involving making a hole in the hood or measuring temperature from the outside. These studies simply compared the skin surface temperature measured by the infrared camera and thermistor. It has a problem that they did not compare at the same body area due to the thermistor generating heat itself. Furthermore, there have been no previous reports about the emissivity of neonates' skin and how it changes with growth. Hence, this study focused on examining and improving the accuracy of measurement of the object by IRT installed in the neonatal incubator before assessment of neonates' body temperature and emissivity. We confirmed that our method had general applicability by examining three commercially available thermal cameras, i.e., FLIR A35 defined as IRT-1 and 2, and FLIR Lepton 3.5 defined as IRT-3.

\section{Conventional Correction Equation and Error Factors of IRT}

As it is dangerous to use a heating element, such as a blackbody furnace, inside a neonatal incubator, we used a non-heating blackbody $\left(\mathrm{BB}_{\mathrm{rs}}\right)$ as a reference source in this study. A blackbody furnace $\left(\mathrm{BB}_{\mathrm{obj}}\right)$ was used as a substitute for the neonate and was not used as a reference source.

When the measurement object is an opaque body, IRT receives the sum of emissions from the object, reflected emissions from ambient sources, and emissions from the atmosphere (Fig. 1). 16, 26, 27, 39, 40

$$
\begin{aligned}
W_{\mathrm{tot}}= & \varepsilon_{\mathrm{obj}} \cdot \tau_{\mathrm{atm}} \cdot W_{\mathrm{obj}}+\left(1-\varepsilon_{\mathrm{obj}}\right) \cdot \tau_{\mathrm{atm}} \cdot W_{\mathrm{ref}} \\
& +\left(1-\tau_{\mathrm{atm}}\right) \cdot W_{\mathrm{atm}}
\end{aligned}
$$

where $\varepsilon_{\mathrm{obj}}$ is the emissivity of the object, $\tau_{\mathrm{atm}}$ is the transmittance of the atmosphere, $W_{\text {obj }}$ is the emission of the object, $W_{\text {ref }}$ is the reflected emission of ambient sources, and $W_{\text {atm }}$ is the emission of the atmosphere. 


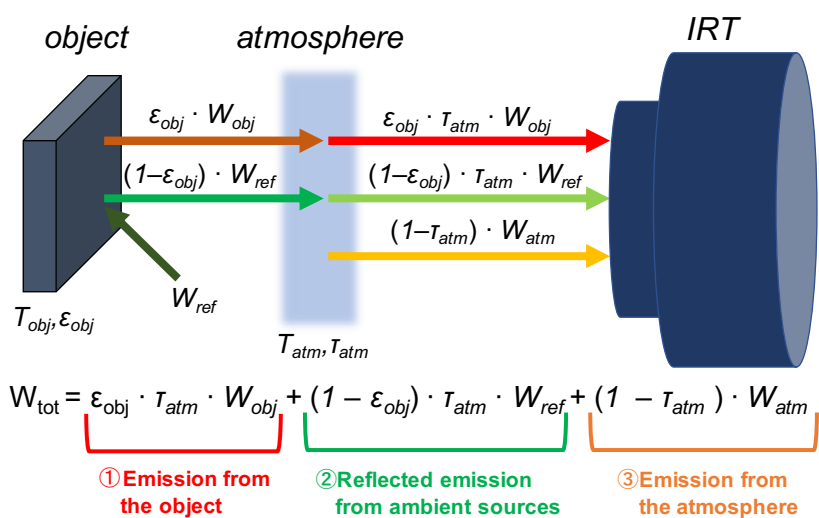

FIGURE 1. Total emission from the object.

Subsequent equation expansion is performed by radiance. Temperature conversion and radiance conversion are performed using the Stefan-Boltzmann equation (2).

$$
\begin{aligned}
W & =\sigma \times T^{4} \\
T & =\sqrt[4]{\frac{W}{\sigma}}
\end{aligned}
$$

where $\sigma$ is the Stefan-Boltzmann constant (equal to $5.670367 \times 10^{-8} \mathrm{~W} / \mathrm{m}^{2} \cdot \mathrm{K}^{4}$ ).

When IRT is used inside the convective neonatal incubator, the emission of the ambient sources $\left(W_{\mathrm{amb}}\right)$ is equal to the emission of the atmosphere $\left(W_{\mathrm{atm}}\right)$ due to the uniform temperature in the incubator. ${ }^{20}$ Therefore, the emission from the object detected by IRT $\left(W_{\text {det1 }}\right)$ can be obtained from Eq. (3):

$$
W_{\mathrm{obj}}=\frac{W_{\mathrm{det} 1}-W_{\mathrm{amb}}}{\varepsilon_{\mathrm{obj}} \tau_{\mathrm{atm}}}+W_{\mathrm{amb}}
$$

Here, the transparency of the atmosphere $\left(\tau_{\text {atm }}\right)$ is calculated from Eqs. (4) and (5). The equations used by the FLIR tools are as follows ${ }^{17,} 39$ :

$$
\begin{aligned}
\tau(d, \omega)= & K_{\mathrm{atm}} \exp \left[-\sqrt{d}\left(\alpha_{1}+\beta_{1} \sqrt{\omega}\right)\right] \\
& +\left(1-K_{\mathrm{atm}}\right) \exp \left[-\sqrt{d}\left(\alpha_{2}+\beta_{2} \sqrt{\omega}\right)\right]
\end{aligned}
$$

$$
\begin{aligned}
\omega\left(\omega_{\%}, T_{\mathrm{atm}}\right)= & \omega_{\%} \\
& \cdot \exp \left(h_{1}+h_{2} \cdot T_{\mathrm{atm}}+h_{3} \cdot T_{\mathrm{atm}}^{2}+h_{4} \cdot T_{\mathrm{atm}}^{3}\right)
\end{aligned}
$$

where $\tau$ is the transmittance of the atmosphere, $K_{\mathrm{atm}}$ is the scaling factor for atmospheric damping (1.9), $d$ is the distance from the IRT, $\alpha_{1}$ and $\alpha_{2}$ are attenuation factors for the atmosphere without water vapor, $\beta_{1}$ and $\beta_{2}$ are attenuation factors for water vapor, $\omega$ is the coefficient indicating the content of water vapor in the atmosphere, $\omega_{\%}$ is the relative humidity, and $h_{1}=$
$1.5587, h_{2}=6.939 \times 10^{-2}, h_{3}=-2.7816 \times 10^{-4}$, and $h_{4}$ $=6.8455 \times 10^{-7}$.

Convective incubators with high temperature and humidity are used in the clinical management of premature infants, and both humidity and ambient temperature are known to affect the measurement of temperature by IRT. ${ }^{3}$ Even conventional IRT software can adjust for these parameters. The error factors of IRT can be divided into two types, i.e., external error factors that are due to the measurement environment and internal error factors that are due to the structure of the IRT device. ${ }^{16}$ The conventional equation (3) is generally used to correct the measurements. However, Eq. (3) takes external error factors into consideration but does not consider the influence of internal error factors. A cooled infrared thermograph cannot be used for IRT inside an incubator as the refrigerant could adversely affect the condition of the neonate. Furthermore, despite their advantages in terms of size and cost, the accuracy of uncooled IRTs is known to be inadequate. ${ }^{31,34}$ Therefore, the use of an uncooled IRT requires the incorporation of internal errors, including emissions from the body of the IRT and the temperature characteristics of the sensor, into body temperature calculations. ${ }^{16}$ To cope with such errors associated with emissions from the body of the IRT that manifest as thermal drift of the focal plane array (FPA) ${ }^{10,}{ }^{24}$ an uncooled IRT calibrates the temperature using a mechanical shutter during non-uniformity correction (NUC). To achieve high accuracy, calibration is required every time the incubator settings are changed to deal with errors associated with the temperature characteristics of the sensor. However, we found that IRT yields inaccurate measurements even with appropriate adjustment of all of the software parameters because NUC operation is intermittent. Standard two-point calibration cannot completely eliminate the influences of thermal drift as they fluctuate over time. In this study, a reference source was used based on the concept that both the measured object and a reference source would be equally influenced by thermal drift. Curcio and Haberman ${ }^{14}$ reported that IRT cannot accurately recognize temperature differences, and many groups have used an independent thermal reference system to improve the accuracy of measurements. ${ }^{12}$ A screening thermograph consists of an IRT and an external temperature reference source (ETRS). ${ }^{19},{ }^{21}$ Simpson et al. ${ }^{37}$ reported that the National Physical Laboratory (NPL) developed a multi-fixed-point source as an in-image calibration system. However, this system was not used in the present study because it was difficult to use inside the incubator. Therefore, a blackbody was used as a reference source the temperature of which was affected by the incubator settings, but it was not harmful when 
used inside the incubator due to its passive thermal control.

\section{Establishment of a Comparison Equation}

The emission from the blackbody furnace $\left(\mathrm{BB}_{\mathrm{obj}}\right)$ as a measured object and the blackbody $\left(\mathrm{BB}_{\mathrm{rs}}\right)$ as a reference source were measured simultaneously (Fig. 2). The estimated emission of $\mathrm{BB}_{\mathrm{rs}}\left(W_{\mathrm{rs}}\right)$ was obtained from equation (6):

$$
W_{\mathrm{bb}}=\frac{W_{\mathrm{det} 2}-W_{\mathrm{amb}}}{\varepsilon_{\mathrm{rs}} \cdot \tau_{\mathrm{atm}}}+W_{\mathrm{amb}}
$$

where $\varepsilon_{\mathrm{rs}}$ is the emissivity of $\mathrm{BB}_{\mathrm{rs}}$ and $W_{\mathrm{det} 2}$ is the emission from $\mathrm{BB}_{\mathrm{rs}}$ detected by the IRT.

A comparison equation was established as correction equation (7) calculated from the internal emission of $\mathrm{BB}_{\mathrm{rs}}$ measured by the thermistor ( $\left.W_{\mathrm{TMT}-\mathrm{rs}}\right)$ and the difference between equations (3) and (6):

$$
\begin{aligned}
W_{\mathrm{COR} 1} & =W_{\mathrm{obj}}-W_{\mathrm{rs}}+W_{\mathrm{TMT}-\mathrm{rs}} \\
& =\frac{W_{\mathrm{det} 1}-W_{\mathrm{amb}}}{\varepsilon_{\mathrm{obj}} \cdot \tau_{\mathrm{atm}}}-\frac{W_{\mathrm{det} 2}-W_{\mathrm{amb}}}{\varepsilon_{\mathrm{rs}} \cdot \tau_{\mathrm{atm}}}+W_{\mathrm{TMT}-\mathrm{rs}}
\end{aligned}
$$

where $W_{\text {TMT-rs }}$ is the emission of the blackbody measured by the thermistor.

In a steady state, the emission from $\mathrm{BB}_{\mathrm{rs}}\left(W_{\mathrm{det} 2}\right)$ equilibrates with the emission of the ambient sources inside the incubator $\left(W_{\mathrm{amb}}\right)$. Therefore, $W_{\mathrm{amb}}$ can be expressed as $W_{\text {det2 }}$ :

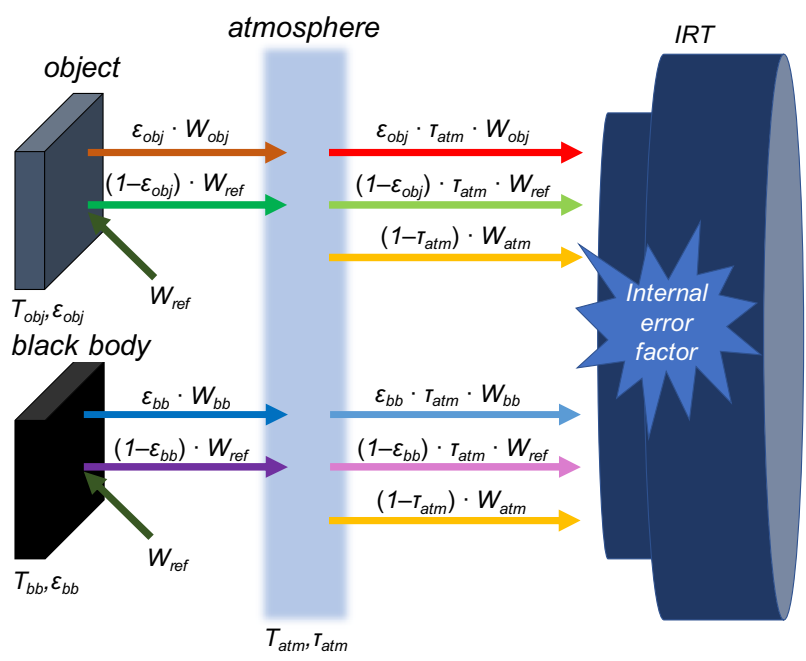

FIGURE 2. Total emissions from the object $\left(B B_{o j}\right)$ and the reference source $\left(\mathrm{BB}_{\mathrm{rs}}\right)$.

$$
\begin{aligned}
W_{\mathrm{COR} 1} & =\frac{W_{\mathrm{det} 1}-W_{\mathrm{det} 2}}{\varepsilon_{\mathrm{obj}} \cdot \tau_{\mathrm{atm}}}-\frac{W_{\mathrm{det} 2}-W_{\mathrm{det} 2}}{\varepsilon_{\mathrm{rs}} \cdot \tau_{\mathrm{atm}}}+W_{\mathrm{TMT}-\mathrm{rs}} \\
& =\frac{W_{\mathrm{det} 1}-W_{\mathrm{det} 2}}{\varepsilon_{\mathrm{obj}} \cdot \tau_{\mathrm{atm}}}+W_{\mathrm{TMT}-\mathrm{rs}}
\end{aligned}
$$

When using a single-detector scanning camera, all points have identical parameters as they are all measured by the same detector. ${ }^{25}$ These values also include internal error, so not only external error but also internal error can be taken into consideration when using Eq. (8).

\section{Establishment of a Linear Equation}

Mean absolute error (MAE) is defined as the mean of the difference in absolute values between the value calculated by each correction equation and the real temperature of the blackbody furnace $\left(\mathrm{BB}_{\mathrm{obj}}\right)$.

When using Eq. (8), the MAE tended to increase when differences between the temperature inside the incubator and the temperature of $\mathrm{BB}_{\text {obj }}$ were large. Moreover, these tendencies differed between individual cameras. Furthermore, relative humidity inside the incubator had no influence on MAE (Fig. 3). Therefore, we postulated that the measurement accuracy would be improved by using regression analysis:

$$
W_{C O R 2}=a \cdot\left(\frac{W_{\mathrm{det} 1}-W_{\mathrm{det} 2}}{\varepsilon_{\mathrm{obj}} \cdot \tau_{\mathrm{atm}}}\right)+W_{\mathrm{TMT}-\mathrm{rs}}+b
$$

where $a$ and $b$ are the regression coefficients.

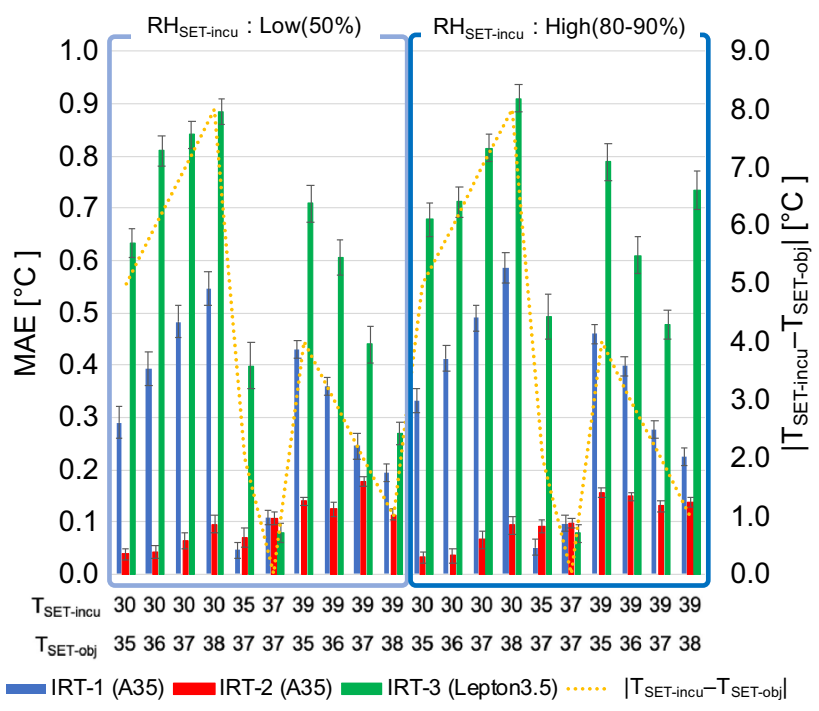

FIGURE 3. Differences in MAE under each set of conditions when corrected by Eq. (8). MAE of $T_{\text {COR1 }}$ are shown for each condition and each camera. The error bars show the standard deviation. The yellow dashed line indicates the absolute value of difference between $T_{\mathrm{SET} \text {-obj }}$ and $T_{\mathrm{SET} \text {-incu. The conditions }}$ were divided into two groups according to the relative humidity (Low: 50\%; High: $80 \%-90 \%$ ). 
Regression analysis was performed using Eq. (9) and the data of Appendix Table S1-S3 and the values of the parameters were as follows: (IRT-1) $a$ : 0.924892, $b: 0.0704166, R^{2}$ : 0.999931; (IRT-2) $a: 0.9805749, b$ : $0.0821071, R^{2}: 0.99997$; (IRT-3) $a: 1.1669497, b$ : -0.075513 , and $R^{2}: 0.998043$.

Moreover, while equation (9) showed good performance with regression analysis using 24 conditions, equation (10) showed good performance with the number of conditions reduced to two selected where the difference between the object to be measured ( $T_{\mathrm{SET}}$ $\left.{ }_{\text {obj }}\right)$ and the incubator temperature $\left(T_{\mathrm{SET} \text {-incu }}\right)$ was large: $T_{\text {SET-incu }}: 30^{\circ} \mathrm{C}, T_{\text {SET-obj }}: 38^{\circ} \mathrm{C}$; and $T_{\text {SET-incu }}$ : $\left.39^{\circ} \mathrm{C}, T_{\text {SET-obj: }}: 35^{\circ} \mathrm{C}\right)$ :

$$
W_{\mathrm{COR} 2}^{\prime}=a^{\prime} \cdot\left(\frac{W_{\mathrm{det} 1}-W_{\mathrm{det} 2}}{\varepsilon_{\mathrm{obj}} \cdot \tau_{\mathrm{atm}}}\right)+W_{\mathrm{TMT}-\mathrm{rs}}+b^{\prime}
$$

where $a^{\prime}$ and $b^{\prime}$ are the regression coefficients. The values of the parameters were as follows: (IRT-1) $a^{\prime}$ : 0.9267647, $b^{\prime}:$ 0.0649386; (IRT-2) $a^{\prime}: 0.9815677, b^{\prime}$ : 0.0555107 ; (IRT-3) $a^{\prime}: 1.1510959, b^{\prime}:-0.161881$.

(a)

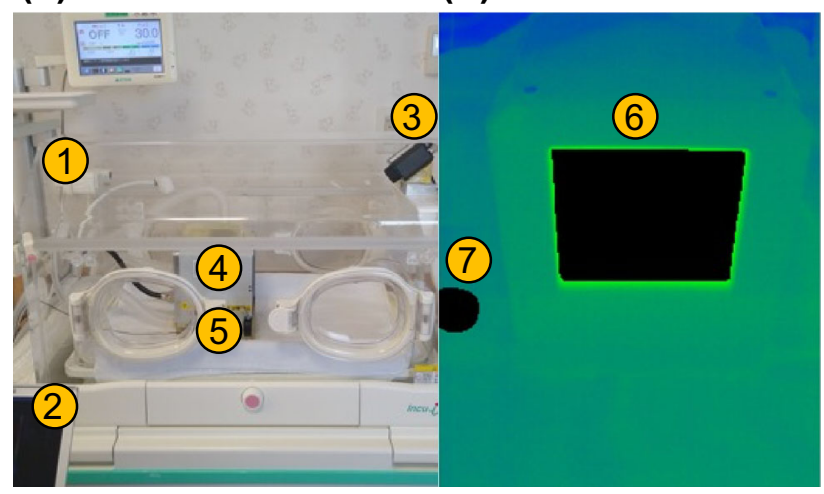

FIGURE 4. Incubator settings and thermal images of the IRT accuracy experiment. (a) IRT imaging inside the incubator: (1) convective neonatal incubator; (2) analysis workstation; (3) infrared thermography camera; (4) blackbody furnace $(\varepsilon=$ $0.97)$; (5) blackbody $(\varepsilon=0.95)$. (b) IRT image of the blackbody furnace and the blackbody: (6) trace area of the blackbody furnace; (7) trace area of the blackbody.

\section{MATERIALS AND METHODS}

The IRT was installed inside a convective neonatal incubator (Incu i; Atom Medical Corporation, Tokyo, Japan) and the temperature of the blackbody furnace $\left(\mathrm{BB}_{\mathrm{obj}}\right)$ was determined as the measured object (Fig. 4). The technical specifications of the blackbody furnace are listed in Table 1.

The uncooled microbolometers IRT-1 and IRT-2 (A35; FLIR Systems Inc., Portland, OR, USA) and IRT-3 (Lepton 3.5; FLIR Systems Inc.) were used in this study. The technical specifications of the IRTs are listed in Table 2.

A35 with two cameras (IRT-1, IRT-2) was used to examine the differences within the same model, and a smaller power-saving model, Lepton 3.5 (IRT-3), was also used to compare the differences between machines. FLIR tools were used to analyze the results obtained with A35, while an analysis tool developed in house was used to analyze the results obtained with Lepton 3.5. In this study, the emissivity of the object was set to 1.0 to obtain the total emission from the object. A standard two-point calibration was not performed beforehand.

All exhaust gas from $\mathrm{BB}_{\mathrm{obj}}$ was emitted to the outside of the incubator through the circuit so that the temperature and humidity inside the incubator were unaffected by the gas. As a reference source, a blackbody $\left(\mathrm{BB}_{\mathrm{rs}}\right) 3.8 \mathrm{~cm}$ in diameter and $1.5 \mathrm{~cm}$ in height, wrapped with tape of emissivity 0.95 , was installed inside the same FOV. The thermistor (19X18-01; Nikkiso-Therm Co., Ltd., Tokyo, Japan) was placed inside the hole at the center of $\mathrm{BB}_{\mathrm{rs}}$ to measure its internal temperature and was connected to a data logger (N543; Nikkiso-Therm Co., Ltd.). BB obj $_{\text {and }}$ $\mathrm{BB}_{\mathrm{rs}}$ were installed at the same distances from the IRT. To minimize the reflected radiation from the surroundings, the mattress was covered with a low-reflectance cloth commonly used for neonates. Relative humidity $\left(R H_{\text {SET-incu }}\right)$ and temperature inside the incubator $\left(T_{\text {SET-incu }}\right)$ were measured using the sensor of the incubator and were maintained at $30-39^{\circ} \mathrm{C}$ and $50 \%-90 \%$, respectively. BB-1 temperatures $\left(T_{\text {SET-obj }}\right)$ were set to $35-38^{\circ} \mathrm{C}$, which are widely accepted as within normal limits for neonates. The experiments were conducted under 24 different settings corresponding to common settings used for incubator

TABLE 1. Technical data of blackbody furnace

\begin{tabular}{|c|c|c|c|c|c|c|}
\hline Product name & Caliber [inches] & Temperature resolution $\left[{ }^{\circ} \mathrm{C}\right]$ & Uniformity $\left[{ }^{\circ} \mathrm{C}\right]$ & Accuracy $\left[{ }^{\circ} \mathrm{C}\right]$ & Stability $\left[{ }^{\circ} \mathrm{C}\right]$ & Emissivity \\
\hline SR800N-4A & $4 \times 4$ & 0.001 & \pm 0.01 & $\begin{array}{l} \pm 0.015 @ \Delta T<0 \\
\pm 0.007 @ 0<T<50 \\
\pm 0.015 @ \Delta T>50\end{array}$ & $\begin{array}{l} \pm 0.003 \\
@ \Delta T< \pm 10\end{array}$ & $0.97 \pm 0.02$ \\
\hline
\end{tabular}


TABLE 2. Technical data of IRT

\begin{tabular}{|c|c|c|c|c|c|c|}
\hline $\begin{array}{l}\text { Product } \\
\text { name }\end{array}$ & $\begin{array}{l}\text { Spectral range } \\
{[\mu \mathrm{m}]}\end{array}$ & $\begin{array}{l}\text { Operating ran- } \\
\text { ge }\left[{ }^{\circ} \mathrm{C}\right]\end{array}$ & $\begin{array}{c}\text { Thermal sensitivity/ } \\
\text { NETD }\end{array}$ & $\begin{array}{l}\text { Image sensor dimensions } \\
\text { (pixels) }\end{array}$ & $\begin{array}{l}\text { Detector pitch } \\
(\mu \mathrm{m})\end{array}$ & Accuracy \\
\hline A35 & $7.5-13$ & $-25 \pm 100$ & $\begin{array}{l}<0.05^{\circ} \mathrm{C} @ 30^{\circ} \mathrm{C} \\
/ 50 \mathrm{mK}\end{array}$ & $320 \times 256$ & 17 & $\pm 5^{\circ} \mathrm{C}$ or $\pm 5 \%$ \\
\hline Lepton3.5 & $8-14$ & $-10 \pm 140$ & $\begin{array}{l}<0.05{ }^{\circ} \mathrm{C} @ 30^{\circ} \mathrm{C} \\
/ 50 \mathrm{mK}\end{array}$ & $160 \times 120$ & 12 & $\pm 5^{\circ} \mathrm{C}$ or $\pm 5 \%$ \\
\hline
\end{tabular}

management to examine their effects on measurement accuracy.

After confirming the stability of the temperature inside $\mathrm{BB}_{\mathrm{rs}}$ under each condition, temperature data of the IRT were captured for 20 minutes under each condition. The data were obtained at a rate of once per second yielding a total of 1200 data points. The temperatures of $\mathrm{BB}_{\mathrm{obj}}$ and $\mathrm{BB}_{\mathrm{rs}}$ were obtained simultaneously from each image (Fig. 1b). Then, the top $10 \%$ upper and lower values were omitted and the average values for each image were calculated. Similarly, the average values were calculated for each condition.

All data were analyzed using JMP ${ }^{\circledR}$ Pro (Ver. 16.0.0; SAS Institute Inc., Cary, NC, USA). MAE was calculated each second under each condition and the average values were compared. When calculating MAE, the setting temperature of $\mathrm{BB}_{\text {obj }}$ was used as the real temperature because the furnace has high accuracy $\left( \pm 0.007{ }^{\circ} \mathrm{C}\right)$ and stability $\left( \pm 0.003{ }^{\circ} \mathrm{C}\right)$. The coefficients of equations (9) and (10) were derived from the results obtained with Eq. (8) by regression analysis. Furthermore, the estimated values obtained with Eqs. (8), (9), and (10) were compared to the conventional equation (3). ASTM E1965-98 ${ }^{7}$ specifies that the accuracy of IRT for use as a skin thermometer must be $<0.3{ }^{\circ} \mathrm{C}$ and IEC 80601-2-59 ${ }^{21}$ specifies that the stability of IRT for use in fever screening must be $<0.1$ ${ }^{\circ} \mathrm{C}$. Therefore, we defined high accuracy and high stability as MAE $<0.3{ }^{\circ} \mathrm{C}$ and standard deviation $<$ $0.1{ }^{\circ} \mathrm{C}$, respectively, where MAE is defined as the mean absolute error between the values estimated by Eqs. (3), (8), (9), and (10) and the actual temperature. Accuracy was evaluated as the percentage of the number of conditions within $\pm 0.3{ }^{\circ} \mathrm{C}$ of the set temperature of BB-1. Using the average of 72 conditions (with three cameras, each camera was investigated under 24 conditions), the accuracy of each equation was evaluated by the $\chi^{2}$ test. A two-tailed $P<0.05$ was taken to indicate statistical significance.

\section{RESULTS}

The dataset in this article presents the MAE calculated using Eqs. (3), (8), (9), and (10) (Appendix Table S1-S3). The MAE of IRT-2 and IRT-3 were high with the conventional equation (3). Using Eq. (3), the MAE of IRT-3 exceeded $3{ }^{\circ} \mathrm{C}$ in some cases, and the MAE values were different for IRT-1 and IRT-2 even with the same specifications.

The differences between each condition and MAE for each camera are shown in Fig. 5. The blackbody furnace $\left(T_{\text {SET-obj }}\right)$ was set to temperatures of $35,36,37$, and $38^{\circ} \mathrm{C}$. The solid line indicates the complete match with the ideal value and the two dashed lines indicate the range $\pm 0.3^{\circ} \mathrm{C}$. Blue points indicate those within \pm $0.3{ }^{\circ} \mathrm{C}$ accuracy, while red points indicate those outside $\pm 0.3{ }^{\circ} \mathrm{C}$. Numbers on the lower right indicate the proportion within $\pm 0.3{ }^{\circ} \mathrm{C}$ of the ideal temperature. The top row "(a)" was calculated using equation (3) as $T_{\text {obj, }}$, the second row "(b)" was calculated using equation (8) as $T_{\mathrm{COR} 1}$, the third row "(c)" was calculated using equation (9) as $T_{\mathrm{COR} 2}$, and the bottom row "(d)" was calculated using equation (10) as $T_{\text {COR2 }}^{\prime}$.

The distances were limited as IRT was installed inside the incubator, and there were only small differences in the values of $\tau$ under each condition ( $\tau$ range: 0.9920-0.9948).

The accuracies of the cameras are shown in Fig. 5. Using the conventional Eq. (3), $T_{\text {obj }}$ tended to be higher than the ideal value (Fig. 5(a)). The standard deviation was still high despite using a relatively accurate camera, such as IRT-1 (Appendix Table S1). Equation (8) was shown to have significantly higher accuracy than Eq. (3) $\left(54.2 \%\right.$ vs. $4.2 \%$, respectively; $\chi^{2}$ =43.6, $P<0.0001$ ) (Figs. 5(a), (b)). However, the error was affected by the measurement conditions, and values of $T_{\mathrm{COR} 1}$ fluctuated depending on the measurement conditions even with $\mathrm{BB}_{\mathrm{obj}}$ set to the same temperature. In addition, the error varied between cameras. Therefore, accurate values were estimated using Eq. (9) as it can offset the variation in measurement conditions and had significantly higher accuracy than Eq. (8) (97.2 vs. 54.2\%, respectively; $\chi^{2}$ $=36.3, P<0.0001)$ (Fig. 5(c)).

Furthermore, Eq. (10) had significantly higher accuracy than Eq. (3) (93.1 vs. $4.2 \%$, respectively; $\chi^{2}=$ 113.9, $P<0.0001$ ) (Fig. 5(d)), and there was no significant difference in accuracy between Eqs. (9) and (10) $\left(\chi^{2}=1.4, P=0.25\right)$. 


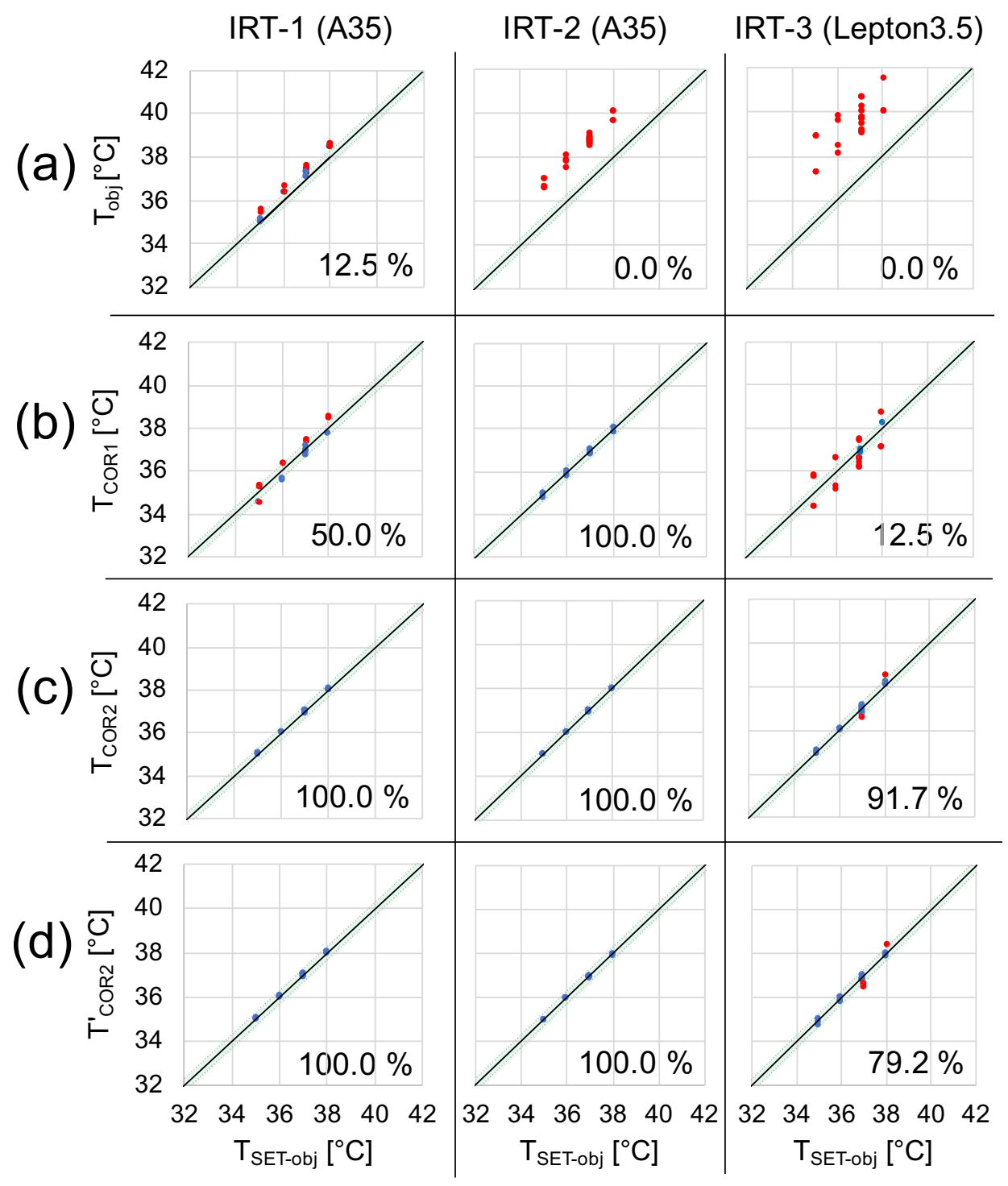

FIGURE 5. Accuracy of each correction equation and the proportion within $\pm 0.3^{\circ} \mathrm{C}$ of the ideal temperature.

\section{DISCUSSION}

Previous studies have measured the body temperature of neonates inside the incubator by IRT 2. ${ }^{1,2,22,}$ 23, 29 In these studies, the temperature measured by IRT was compared to that determined with a thermistor as the contact temperature. However, in premature infants, the contact measurement method is also inaccurate due to characteristics of the premature skin. ${ }^{22,}{ }^{35}$ Furthermore, previous studies on the effectiveness and accuracy of IRT applied to newborn infants were mostly conducted with the IRT placed outside the incubator, and there have been no reports on the accuracy of IRT installed inside the incubator. Therefore, we assessed the measurement accuracy of the IRT installed inside an incubator using a blackbody furnace.

The conventional Eq. (3) eliminates the external error factors, but there were conditions under which the error exceeded $10 \%$ (IRT-3, $T_{\mathrm{SET} \text {-incu }}: 39^{\circ} \mathrm{C}, T_{\mathrm{SET}-}$ obj: $38^{\circ} \mathrm{C}, R H_{\text {SET-incu: }}$ : $80 \%$ ) (Appendix Table S3). Equation (8) can also eliminate internal error factors because it uses the same FOV temperature differences. However, the differences between individual cameras as well as the differences in temperature between $\mathrm{BB}_{\mathrm{obj}}$ and internal environment of the incubator also had an influence. Therefore, Eq. (9) used regression analysis and determined the coefficients of each camera, which resulted in improved accuracy. Moreover, Eq. (10) 


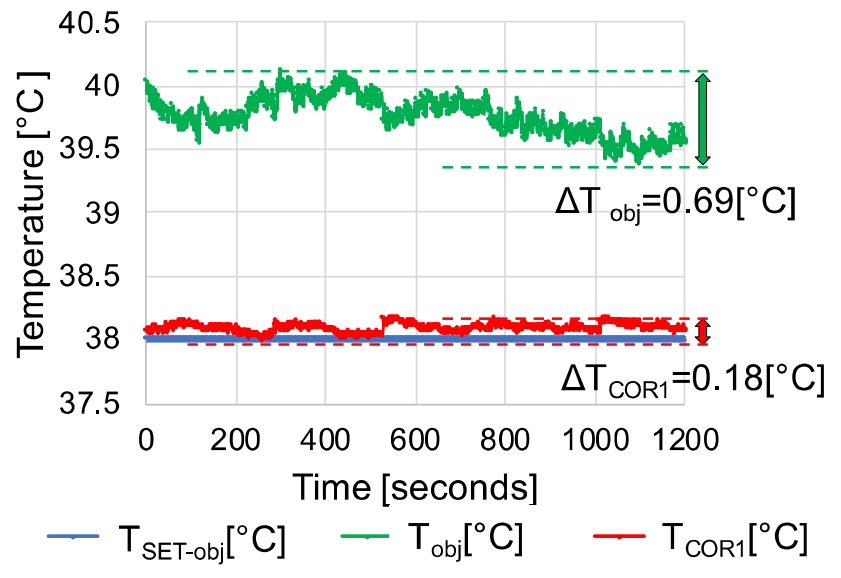

FIGURE 6. Comparison of $T_{\text {obj }}$ and $T_{\text {COR1 }}$ over time with the corrected data using the conventional equation (3): $T_{\text {obj }}$, and using equation (8): $T_{\text {COR1 }}$ (IRT-2, settings $T_{\mathrm{SET}-\text { incu }}: 30^{\circ} \mathrm{C}, T_{\mathrm{SET}}$ obj: $38^{\circ} \mathrm{C}, \mathrm{RH}_{\mathrm{SET} \text {-incu: }}$ : $90 \%$ )

showed high accuracy even with reduction of the conditions from 24 to only 2 .

Figure 6 shows that the values obtained with equation (3) were inaccurate regardless of the calibration phase using a mechanical shutter. Abbas et al. ${ }^{1}$ limited the recording time in their study due to the IRT recalibration phases. In this study, however, the errors were large even with limitation of the measurement phases. In these cases, standard two-point calibrations are required. However, the influences of temperature changes, not only of the neonate but also of the conditions inside the incubator, must be taken into consideration when applying IRT in an incubator. Therefore, frequent calibrations are required whenever the settings of the incubator are changed. ${ }^{10}$

Furthermore, even when using the same equipment, MAE differs regardless of the noise equivalent temperature difference (NETD) and signal transfer function (SiTF). Minkina and Dudzik ${ }^{25}$ reported that the accuracy of IRT is dependent on calibration by the manufacturer. In addition, Cao and Tisse ${ }^{10}$ and Riou et al $^{33}$ reported that outputs were different between individual detectors even when using the same equipment. In fact, the accuracy differed between detectors even using the same equipment in the present study (Fig. 5(a)). Therefore, it is necessary for manufacturers to adjust the individual equipment because most types of IRT have a wide operating range. Moreover, even if it were possible to calibrate for a narrow operating range, there would still be issues associated with thermal drift. Riou et al..$^{33}$ reported that improvement of the accuracy of thermal infrared cameras required correction for thermal drift. Therefore, we adopted the relative temperature of the IRT in the present study based on the concept that all points had identical parameters as the measurements were obtained with the same detector. ${ }^{25}$

Our method achieved high accuracy. The conventional equation (3) showed fluctuation over time of $0.69{ }^{\circ} \mathrm{C}$, while this was decreased to $0.18{ }^{\circ} \mathrm{C}$ with the use of Eq. (8) (Fig. 6). However, appropriate corrections could be made in some cases, but this was not possible in other cases. Even using equation (8), the influence of the incubator settings could not be eliminated (Fig. 5(b)), and there were differences in estimated values even when using the same blackbody furnace settings. Both Eqs. (3) and (8) incorporated the environmental temperature, but its influence could not be entirely eliminated. Furthermore, the mechanical shutter calibration process depends on information regarding temperature inside the housing of the IRT, and was also not sufficient to eliminate the influence of the environment. Therefore, adjustments were made by regression analysis. Equation (9) was affected by the temperature difference between the incubator settings and the object to be measured, as well as by the differences between cameras. Therefore, each camera was adjusted by regression analysis, which made it possible to measure temperatures with high accuracy at all incubator settings (Fig. 5(c)).

This calibration method takes both the measurement environment temperature and the temperature range of the object to be measured into consideration. In contrast to existing multipoint calibration methods that only consider the temperature of the object to be measured, adjustments can be made with the temperature of the incubator and the temperature range of the object to be measured. Furthermore, multipoint calibration cannot be performed with a neonate in the incubator as it requires the use of a dangerous heat source. However, a calibration source is required to deal with thermal drift.

In this study, the regression equation was initially obtained using 24 conditions for each camera, and we then confirmed that the number of conditions could be reduced to only two. Therefore, analysis of the results of these two conditions and determination of the coefficient beforehand will allow accurate measurements in other situations. However, there were differences in accuracy between IRT-1, IRT-2, and IRT-3. All conditions of IRT- 1 and IRT-2 had accuracies \pm $0.1{ }^{\circ} \mathrm{C}$, whereas $20.8 \%$ of IRT-3 conditions were outside of $\pm 0.3^{\circ} \mathrm{C}$ even when using Eq. (9). Although the accuracy was the same for both A35 and Lepton 3.5, we still feel that there are limitations when using the smaller power-saving model (Table 2).

As shown in the study dataset, the standard deviation of MAE in most conditions exceeded $0.1{ }^{\circ} \mathrm{C}$ even when using the conventional Eq. (3), while Eqs. (9) and (10) achieved MAE standard deviation $<0.1{ }^{\circ} \mathrm{C}$ ex- 
cept for one condition (Appendix Table S1-S3), thus satisfying the requirements of IEC 80601-2-59.21

The high accuracy of thermography with this method is very important for the clinical use of thermography, but it is also important to capture the contours of the neonate in clinical practice. In other words, a method automatically tracking the defined ROI (region of interest) is needed in monitoring the body temperature of neonates. ${ }^{3}$ The neonatal body surface is small and sometimes intertwined with medical cables. Therefore, inaccurate detection of ROI leads to misanalysis of body temperature. Hence, we reported a method for the segmentation of thermal images that enables continuous non-invasive monitoring of the body temperature distribution over the whole body of neonates. ${ }^{6}$ The newly established equation (10) will give new evidence in this field combined with this segmentation method.

In conclusion, the Eq. (10) significantly improved the accuracy and stability of temperature measurements of subjects placed in an incubator. This study will facilitate the development of novel means of administrating neonatal body temperature.

\section{SUPPLEMENTARY INFORMATION}

The online version contains supplementary material available at https://doi.org/10.1007/s10439-022-02937w.

\section{ACKNOWLEDGMENTS}

The authors thank Masaaki Ohashi and Yuto Asayama, Department of Technology Development, Atom Medical Corporation, Saitama, Japan, who prepared the IRT and set the blackbody furnace inside the incubator. The workstation, IRT, software, blackbody furnace, and blackbody used in this study were provided by Atom Medical Corporation.

\section{FUNDING}

Funding was provided by Atom Medical Corporation through the joint research agreement with Atom Medical Corporation, Nagasaki Harbor Medical Center, Kagoshima City Hospital, and Osaka University.

\section{CONFLICT OF INTEREST}

$\mathrm{KH}, \mathrm{EH}, \mathrm{TM}$, TI and YN declare no conflict of interest. No benefits in any form have been or will be received from a commercial party related directly or indirectly to the subject of this manuscript. HA and $\mathrm{HH}$ are salaried employees of Atom Medical Corporation.

\section{OPEN ACCESS}

This article is licensed under a Creative Commons Attribution 4.0 International License, which permits use, sharing, adaptation, distribution and reproduction in any medium or format, as long as you give appropriate credit to the original author(s) and the source, provide a link to the Creative Commons licence, and indicate if changes were made. The images or other third party material in this article are included in the article's Creative Commons licence, unless indicated otherwise in a credit line to the material. If material is not included in the article's Creative Commons licence and your intended use is not permitted by statutory regulation or exceeds the permitted use, you will need to obtain permission directly from the copyright holder. To view a copy of this licence, visit http://crea tivecommons.org/licenses/by/4.0/.

\section{REFERENCES}

${ }^{1}$ Abbas, A. K., K. Heimann, K. Jergus, T. Orlikowsky, and S. Leonhardt. Neonatal non-contact respiratory monitoring based on real-time infrared thermography. Biomed. Eng. Online. 10:93, 2011. https://doi.org/10.1186/1475-925 $\mathrm{x}-10-93$

${ }^{2}$ Abbas, A. K., K. Heimann, V. Blazek, T. Orlikowsky, and S. Leonhardt. Neonatal infrared thermography imaging: Analysis of heat flux during different clinical scenarios. Phys. Technol. 55:538-548, 2012. https://doi.org/10.1016/j. infrared.2012.07.001.

${ }^{3}$ Abbas, A. K., and S. Leonhardt. Intelligent neonatal monitoring based on a virtual thermal sensor. BMC Med. Imaging. 14:9, 2014. https://doi.org/10.1186/1471-2342-149.

${ }^{4}$ Adamson, S. K., and M. E. Jr. Towell. Thermal homeostasis in the fetus and newborn. Anesthesiology. 26:531548, 1965. https://doi.org/10.1097/00000542-196507000-00 017.

${ }^{5}$ Aggarwal, N., M. Garg, V. Dwarakanathan, N. Gautam, S. S. Kumar, R. S. Jadon, M. Gupta, and A. Ray. Diagnostic accuracy of non-contact infrared thermometers and thermal scanners: a systematic review and meta-analysis. $J$. Travel Med. 2020. https://doi.org/10.1093/jtm/taaa193.

${ }^{6}$ Asano, H., E. Hirakawa, H. Hayashi, K. Hamada, Y. Asayama, M. Oohashi, A. Uchiyama, and T. Higashino. A method for improving semantic segmentation using thermographic images in infants. BMC Med Imaging. 22(1):1, 2022. https://doi.org/10.1186/s12880-021-00730-0.

${ }^{7}$ ASTM International. ASTM E1965-98 Standard Specification for Infrared Thermometers for Intermittent Determination of Patient Temperature. West Conshohocken, Pennsylvania: ASTM International, 2016. 
${ }^{8} \mathrm{Aw}$, J. The non-contact handheld cutaneous infra-red thermometer for fever screening during the COVID-19 global emergency. J. Hosp. Infect. 104:451, 2020. https://d oi.org/10.1016/j.jhin.2020.02.010.

${ }^{9}$ Bell, D. M., WHO. Public health interventions and SARS spread. Emerg. Infect. Dis. 10:1900-1906, 2004. https://doi. org/10.3201/eid1011.040729.

${ }^{10} \mathrm{CaO}$, Y., and C. L. Tisse. Shutterless solution for simultaneous focal plane array temperature estimation and nonuniformity correction in uncooled long-wave infrared camera. Appl. Opt. 52:6266-6271, 2013. https://doi.org/10. 1364/ao.52.006266.

${ }^{11}$ Centers for Disease Control and Prevention. Non-Contact Temperature Measurement Devices: Considerations for Use in Port of Entry Screening Activities. Centers for Disease Control and Prevention (CDC): London, US, 2014. https://stacks.cdc.gov/view/cdc/24857.

${ }^{12}$ Charles, J. A., and J. E. Francis. Design of a thermal reference system for use in thermography. Rev. Sci. Instrum. 44:291-294, 1973. https://doi.org/10.1063/1.1686109.

${ }^{13}$ Cowling, B. J., L. L. Lau, P. Wu, H. W. Wong, V. J. Fang, S. Riley, and H. Nishiura. Entry screening to delay local transmission of 2009 pandemic influenza A (H1N1). BMC Infect. Dis. 10:82, 2010. https://doi.org/10.1186/1471-233410-82.

${ }^{14}$ Curcio, B. M., and J. A. Haberman. Infrared thermography: a review of current medical application, instrumentation and technique. Radiol. Technol. 42:233-247, 1971.

${ }^{15}$ Ferreira, J. J., L. C. Mendonça, L. A. Nunes, A. C. Andrade Filho, J. R. Rebelatto, and T. F. Salvini. Exerciseassociated thermographic changes in young and elderly subjects. Ann. Biomed. Eng. 36:1420-1427, 2008. https://d oi.org/10.1007/s10439-008-9512-1.

${ }^{16}$ FLIR Systems. The Ultimate Infrared Handbook for R\&D Professionals, In IR Thermography-How It Works. Geneva: FLIR Systems Inc, pp. 2-6, 2012. https://www.flirmed ia.com/MMC/THG/Brochures/T559243/T559243_EN.pdf.

${ }^{17}$ FLIR Systems, FLIR. Toolkit IC2 Dig 16: Developers Guide 1.01 for AGEMA 550, 570, ThermaCAM PM $5 \times 5$ and the ThermoVision Family, Version B, FLIR Publication Number: 557344 ed. Wilsonville, OR: FLIR Systems Inc, p. 54, 2001.

${ }^{18}$ Formenti, D., N. Ludwig, M. Gargano, M. Gondola, N. Dellerma, A. Caumo, and G. Alberti. Thermal imaging of exercise-associated skin temperature changes in trained and untrained female subjects. Ann. Biomed. Eng. 41:863-871, 2013. https://doi.org/10.1007/s10439-012-0718-x.

${ }^{19}$ Ghassemi, P., T. J. Pfefer, J. P. Casamento, R. Simpson, and Q. Wang. Best practices for standardized performance testing of infrared thermographs intended for fever screening. PLoS ONE.13:e0203302, 2018. https://doi.org/ 10.1371/journal.pone.0203302.

${ }^{20}$ IEC/ISO, IEC 60601-2-19. Medical Electrical Equipment-Particular Requirements for the Basic Safety and Essential Performance of Infant Incubators. Geneva: International Organization for Standardization ISO, 2020.

${ }^{21}$ IEC/ISO, IEC 80601-2-59. Medical Electrical Equipment-Particular Requirements for the Basic Safety and Essential Performance of Screening Thermographs for Human Febrile Temperature Screening. Geneva: International Organization for Standardization (ISO), 2017.

${ }^{22}$ Knobel, R. B., B. D. Guenther, and H. E. Rice. Thermoregulation and thermography in neonatal physiology and disease. Biol. Res. Nurs. 13:274-282, 2011. https://doi. org/10.1177/1099800411403467.

${ }^{23}$ Knobel-Dail, R. B., D. Holditch-Davis, R. Sloane, B. D. Guenther, and L. M. Katz. Body temperature in premature infants during the first week of life: Exploration using infrared thermal imaging. J. Therm. Biol. 69:118-123, 2017. h ttps://doi.org/10.1016/j.jtherbio.2017.06.005.

${ }^{24}$ Liang, K., C. Yang, L. Peng, and B. Zhou. Nonuniformity correction based on focal plane array temperature in uncooled long-wave infrared cameras without a shutter. Appl. Opt. 56:884-889, 2017. https://doi.org/10.1364/ao.56.0008 84.

${ }^{25}$ Minkina, W., and S. Dudzik. Infrared thermography: error and uncertainties. In: Measurement Infrared Cameras, edited by H. Budzier, and G. Gerlach. NJ: Wiley, Hoboken, 2009, pp. 29-40.

${ }^{26}$ Musto, M., G. Rotondo, M. De Cesare, A. Del Vecchio, L. Savino, and F. D. Filippis. Error analysis on measurement temperature by means dual-color thermography technique. Measurement. 90:265-277, 2016. https://doi.org/10.1016/j. measurement.2016.04.024.

${ }^{27}$ Nakamura, H. Temperature measurement using infrared thermography and its application to convective heat transfer measurement. J. Heat Transfer Soc. Japan. 54:4754, 2015. http://www.htsj.or.jp/dennetsu/dennetsu54-j.htm 1. [in Japanese].

${ }^{28}$ Nunnally, M. E., R. Jaeschke, G. J. Bellingan, J. Lacroix, B. Mourvillier, G. M. Rodriguez-Vega, S. Rubertsson, T. Vassilakopoulos, C. Weinert, S. Zanotti-Cavazzoni, and T. G. Buchman. Targeted temperature management in critical care: a report and recommendations from five professional societies. Crit. Care Med. 39:1113-1125, 2011. https://doi. org/10.1097/CCM.0b013e318206bab2.

${ }^{29}$ Pomerance, J. J., R. L. Lieberman, and C. T. Ukrainski. Neonatal thermography. Pediatrics. 59:345-351, 1977.

${ }^{30}$ Pusnik, I., and J. Drnovsek. Infrared ear thermometersparameters influencing their reading and accuracy. Physiol. Meas. 26:1075-1084, 2005. https://doi.org/10.1088/0967-3 $334 / 26 / 6 / 016$.

${ }^{31}$ Razani, M., A. Parkhimchyk, and N. Tabatabaei. Lock-in thermography using a cellphone attachment infrared camera. AIP Adv. 2018. https://doi.org/10.1063/1.5021601.

${ }^{32}$ Ring, E. F. The historical development of thermometry and thermal imaging in medicine. J. Med. Eng. Technol. 30:192198, 2006. https://doi.org/10.1088/978-0-7503-1143-4ch1.

${ }^{33}$ Riou, O., S. Berrebi, and P. Bremond. Nonuniformity correction and thermal drift compensation of thermal infrared camera. Defense Sec. 5405:884-889, 2004. https://d oi.org/10.1117/12.547807.

${ }^{34}$ Samadi, N., D. Thapa, M. Salimi, A. Parkhimchyk, and N. Tabatabaei. Low-cost active thermography using cellphone infrared cameras: from early detection of dental caries to quantification of THC in oral fluid. Sci. Rep. 10:7857, 2020. https://doi.org/10.1038/s41598-020-64796-6.

${ }^{35}$ Shaul, D., and H. Steven. Temperature regulation in preterm infants: Role of the skin-environment interface. NeoReviews. 2:282-291, 2001. https://doi.org/10.1542/neo. 2-12-e282.

${ }^{36}$ Silverman, W. A., and J. C. Sinclair. Temperature regulation in the newborn infant. N. Engl. J. Med. 274:92-94, 1966. https://doi.org/10.1056/nejm196601132740207.

${ }^{37}$ Simpson, R., H. Mcevoy, G. Machin, K. Howell, M. Naeem, P. Plassmann, E. F. J. Ring, P. Campbell, C. Song, J. Tavener, and I. Ridley. In-field-of-view thermal image calibration system for medical thermography applications. 
Int. J. Thermophys. 29:1123-1130, 2008. https://doi.org/10. 1007/s10765-008-0393-1.

${ }^{38}$ Sund-Levander, M., C. Forsberg, and L. K. Wahren. Normal oral, rectal, tympanic and axillary body temperature in adult men and women: a systematic literature review. Scand. J. Caring Sci. 16:122-128, 2002. https://doi. org/10.1046/j.1471-6712.2002.00069.x.

${ }^{39}$ Tran, Q. H., D. Han, C. Kang, A. Haldar, and J. Huh. Effects of ambient temperature and relative humidity on subsurface defect detection in concrete structures by active thermal imaging. Sensors (Basel). 2017. https://doi.org/10. $3390 / \mathrm{s} 17081718$.

${ }^{40}$ Usamentiaga, R., P. Venegas, J. Guerediaga, L. Vega, J. Molleda, and F. G. Bulnes. Infrared thermography for temperature measurement and non-destructive testing.
Sensors (Basel). 14:12305-12348, 2014. https://doi.org/10. $3390 / \mathrm{s} 140712305$.

${ }^{41}$ Uxa, F. Thermal control of the newborn. Kangaroo. 3:6768, 1994.

${ }^{42}$ Waldron, S., and R. Mackinnon. Neonatal thermoregulation. Infant. 3:101-104, 2007.

${ }^{43}$ Zontak, A., S. Sideman, O. Verbitsky, and R. Beyar. Dynamic thermography: analysis of hand temperature during exercise. Ann. Biomed. Eng. 26:988-993, 1998. https://doi. org/10.1114/1.33.

Publisher's Note Springer Nature remains neutral with regard to jurisdictional claims in published maps and institutional affiliations. 